\title{
Quadratic Boost Converter: An Analysis with Passive Components Losses
}

\author{
Modou Badiane1, Prince Abdoul Aziz Honadia², Bernard Zouma², Fabé Idrissa Barro ${ }^{*}$ \\ ${ }^{1}$ Department of Physics, Semiconductors and Solar Energy Laboratory, Faculty of Science and Technique, Cheikh Anta Diop \\ University, Dakar, Senegal \\ ${ }^{2}$ Department of Physics, Thermal Renewable Energies Laboratory, Training and Research Unit in Exact and Applied Sciences, \\ Joseph Ki-Zerbo University, Ouagadougou, Burkina Faso \\ Email: ^fabe.barro@ucad.edu.sn
}

How to cite this paper: Badiane, M., Honadia, P.A.A., Zouma, B. and Barro, F.I. (2021) Quadratic Boost Converter: An Analysis with Passive Components Losses. Open Journal of Applied Sciences, 11, 202-215.

https://doi.org/10.4236/ojapps.2021.112014

Received: January 6, 2021

Accepted: February 23, 2021

Published: February 26, 2021

Copyright (C) 2021 by author(s) and Scientific Research Publishing Inc. This work is licensed under the Creative Commons Attribution International License (CC BY 4.0).

http://creativecommons.org/licenses/by/4.0/

\begin{abstract}
The Cascaded Connected Single Switch Quadratic Boost $\left(\mathrm{C}^{2} \mathrm{~S}^{2}-\mathrm{QB}\right)$ is studied first from its ideal model, then with semi-real model taking into account resistive losses through the inductors and the capacitor. The continuous conduction mode equations, describing these different models are established, taking into account losses through passive components. From these equations, the voltage gain and the efficiency are determined for the semi-real model. The voltage gain and the efficiency are then analyzed versus duty cycle and the influence of passive component losses on the performance of the quadratic boost converter is carried out for the semi-real model. It has been showed that the quality of the passive components of a converter plays an important role in the quality of the conversion. However, not all passives components affect the converter in the same way.
\end{abstract}

\section{Keywords}

Quadratic Boost, Losses, Gain Factor, Efficiency

\section{Introduction}

Photovoltaic energy is used for daily consumption purposes; however, the direct current delivered by the panels must be suitable for the majority of appliances that only operate in alternating current. This transformation is done using photovoltaic inverters. The voltage delivered by the main being about $220 \mathrm{~V}$ in West Africa, it would be necessary that the photovoltaic system could deliver as much. Taking into account the losses, we would need a DC voltage of about $300 \mathrm{~V}$ from the panels to convert it into a suitable AC voltage. As a result, the number of 
panels, delivering only 15 - $17 \mathrm{~V}$ each on average, to be put in series to obtain the $300 \mathrm{~V}$ is quite considerable. As we are in the context of miniaturization and cost minimization, the inverters include DC-DC step-up converters to raise the voltage from the panels to average voltage values.

The low-frequency characterization of the DC-DC converters was carried out in 1972 [1] [2]. The work presented in [1] can be considered as the first reference to the modeling of DC-DC converters by means of analytical techniques with average continuous time. In the 1990s, the search for a circuit-oriented modeling methodology led to the suggestion of the use of pulse width modulator (PWM-Switch), a three-terminal structure that was the active switch and the diode, the passive switch for most converters [3]-[8]. Later, a general average state model based on the representation of state variables using Fourier series has been proposed [9]. Reference [10] proposes the application of this method to resonant and PWM converters, substituting switches with dependent sources.

At the beginning of the 21st century, a systematic method (applicable to all conduction modes) to obtain circuit-oriented average models for multi-output DC-DC converters is presented [11]. One of the main functions of the DC-DC converter is to also allow an impedance matching between the source, which are the panels and the load (user).

That is, there are many works in the field of DC-DC converters with the main objective in renewable energies being high voltage gain with maximum efficiency with reasonable duty cycle [12]-[17]. In this field, quadratic boost converters are good candidates but there are still some problems to solve: how to minimize losses? How these losses affect the performance of the converter?

Some studies [14] [15] have highlighted losses due to components; however, the question remains as to the proportion that each of its components has in the total losses.

This work focuses on these problems by studying a Cascaded Connected Single Switch Quadratic Boost $\left(\mathrm{C}^{2} \mathrm{~S}^{2}-\mathrm{QB}\right)$, particularly the losses in the passive components of the converter. Based on an electrical and mathematical modelling of the converter, the main equations governing the converter are presented and the analytical expressions of the voltage gain and conversion efficiency taking into account losses through passive components are determined.

\section{Modeling of the Converter and Mathematical Formulation}

The quadratic boost can practically operate in all the possible different modes of conduction according to the values of the duty cycle ratio and the load. The choice of switching into Continuous Conduction Mode (CCM) or Discontinuous Conduction Mode (DCM) has a direct impact on the performance of the power stage and the crossing frequency of the loop.

Also, particular hypotheses can be expected to cope with the analysis of the of discontinuous conduction mode with variable switching frequency. Indeed, the operation in DCM will generally result in a higher loop bandwidth at the ex- 
pense of less efficiency. The converter sized on the basis of this mode will probably be smaller due to smaller inductances, but the requirements on the capacitance of the output capacitor will be higher [11].

The Cascaded Connected Single Switch Quadratic Boost $\left(\mathrm{C}^{2} \mathrm{~S}^{2}-\mathrm{QB}\right)$ is presented in Figure 1.

It consists of a single mosfet transistor which serves as a switch, three diodes $D_{1}, D_{2}$ and $D_{3}$, two capacitors $C_{1}$ and $C_{2}$, two inductors $L_{1}$ and $L_{2}$, and the load resistance $R$ as can be seen in Figure 1 .

Assuming that all components are ideal and the converter is operating in Continuous Conduction Mode (CCM) [16] as this operating mode is more suited for photovoltaic applications, the basic equations are as follows [17]:

$$
\begin{aligned}
& \frac{\mathrm{di} i_{L_{1}}}{\mathrm{~d} t}=\frac{v_{i n}}{L_{1}}-\frac{v_{C_{1}}}{L_{1}}(1-s) \\
& \frac{\mathrm{d} i_{L_{2}}}{\mathrm{~d} t}=\frac{v_{C_{1}}}{L_{2}}-\frac{v_{C_{2}}}{L_{2}}(1-s) \\
& \frac{\mathrm{d} v_{C_{1}}}{\mathrm{~d} t}=-\frac{i_{L_{2}}}{C_{1}}+\frac{i_{L_{1}}}{C_{1}}(1-s) \\
& \frac{\mathrm{d} v_{C_{2}}}{\mathrm{~d} t}=-\frac{V_{C_{2}}}{R C_{2}}+\frac{i_{L_{2}}}{C_{2}}(1-s)
\end{aligned}
$$

When taking into account resistive losses through inductor and capacitor, the boost converter can be presented as shown in Figure 2:

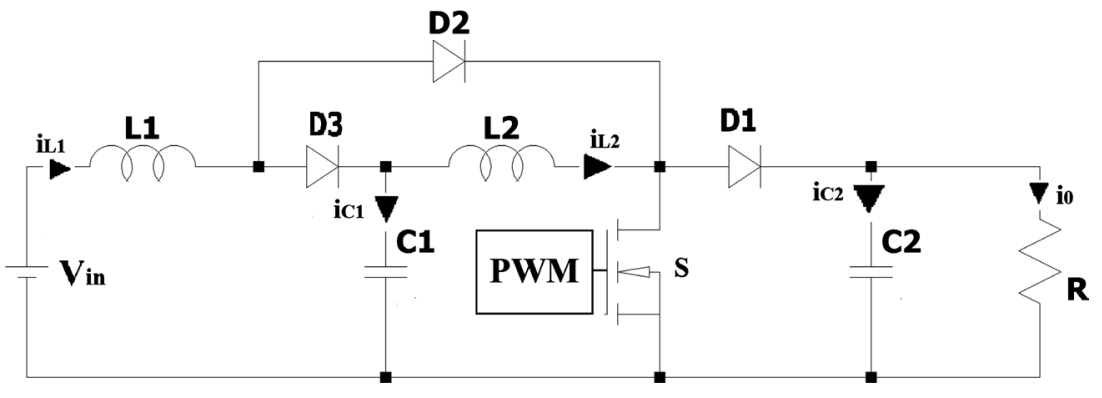

Figure 1. Boost quadratic $\left(\mathrm{C}^{2} \mathrm{~S}^{2}-\mathrm{QB}\right)$.

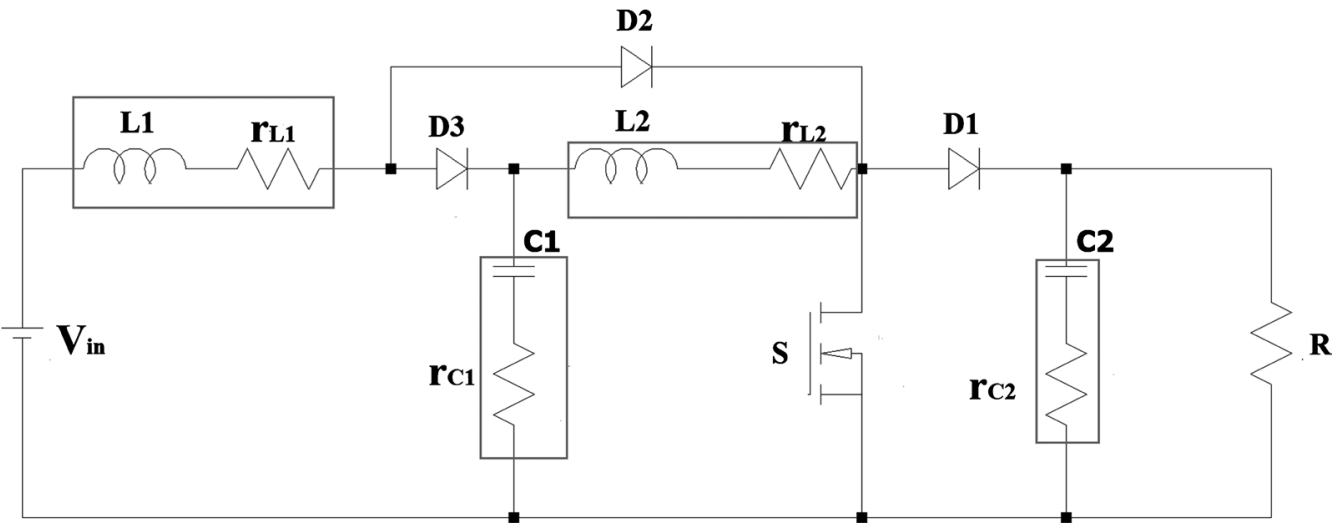

Figure 2. Quadratic boost converter with losses through inductor and capacitor (model 1). 
The power balance is written as:

$$
P_{I}=P_{O}+P_{r_{L_{1}}}+P_{r_{L_{2}}}+P_{r_{C_{1}}}+P_{r_{C_{2}}}
$$

$P_{I}, P_{O}, P_{r_{L_{1}}}, P_{r_{L_{2}}}, P_{r_{C_{1}}}, P_{r_{C_{2}}}$ are respectively the input power, the output power, the power losses through the inductors series resistance $r_{L_{1}}, r_{L_{2}}$ and the power losses through the capacitors series resistance $r_{C_{1}}, r_{C_{2}}$. Expressing the different terms in Equation (5), it can be rewritten in the form:

$$
\begin{aligned}
V_{I} \cdot \frac{I_{0}}{(1-\alpha)^{2}}= & V_{0} \cdot I_{0}+r_{L_{1}} \cdot \frac{I_{0}^{2}}{(1-\alpha)^{4}}+r_{L_{2}} \cdot \frac{I_{0}^{2}}{(1-\alpha)^{2}} \\
& +r_{C_{1}} \cdot \frac{I_{0}^{2} \cdot \alpha(1-\alpha)}{(1-\alpha)^{4}}+r_{C_{2}} \cdot \frac{I_{0}^{2} \cdot \alpha}{(1-\alpha)}
\end{aligned}
$$

We can then derive the gain factor $G$ as:

$$
G=\frac{(1-\alpha)^{2} R}{R(1-\alpha)^{4}+(1-\alpha)^{2} r_{L_{2}}+r_{L_{1}}+\alpha(1-\alpha) r_{C_{1}}+\alpha(1-\alpha)^{3} r_{C_{2}}}
$$

$R$ is the load resistor.

The conversion efficiency $\eta$ is defined by:

$$
\eta=\frac{P_{0}}{P_{0}+P_{L S}}
$$

Replacing $P_{O}, P_{r_{L}}, P_{r_{C}}$ by their respective expressions, the conversion efficiency becomes:

$$
\eta=\frac{1}{1+\left[\frac{\alpha(1-\alpha) r_{C_{1}}+r_{L_{1}}}{(1-\alpha)^{4} R}+\frac{\alpha(1-\alpha) r_{C_{2}}+r_{L_{2}}}{(1-\alpha)^{2} R}\right]}
$$

\section{Results and Discussions}

Based on the mathematical formulation above, we have been able from Mathcad software to plot different voltage gain and Conversion efficiency versus duty cycle. The simulations were performed by varying duty cycle and series resistances, but choosing transistors and diodes as ideals.

\subsection{Voltage Gain Factor}

These curves are intended to highlight and appreciate the inductive and capacitive ESR effects on the quadratic boost performance. The internal resistance, whose effect we want to observe, is parameterized with values ranging from 0.02 $\Omega$ to $0.1 \Omega$ with a pitch of 0.02 . These different values can materialize a progressive deterioration of the component or a use of components of different qualities. However, the values of more than $0.1 \Omega$ are already large enough not to be interested because in practice the use of quality components will be preferred for optimizing the performance of the converter. In the same vein, we will vary the internal resistance of a component assuming that the other components are good qualities, that is to say constant internal resistances and equal to $0.02 \Omega$ 
[18].

Therefore, we present in Figure 3 the voltage gain factor $G$ versus duty cycle $\alpha$ for various inductor series resistance $r_{L_{1}}$ Figure 3(a) and various inductor series resistance $r_{L_{2}}$ Figure 3(b).

These figures show that voltage gain factor first increase until a certain duty cycle $\alpha_{0}$ value from which it began decreasing. In fact, when the duty cycle is increasing the losses in the series resistance of the inductor also increase to a threshold from which the losses are so important that the voltage gain factor begin

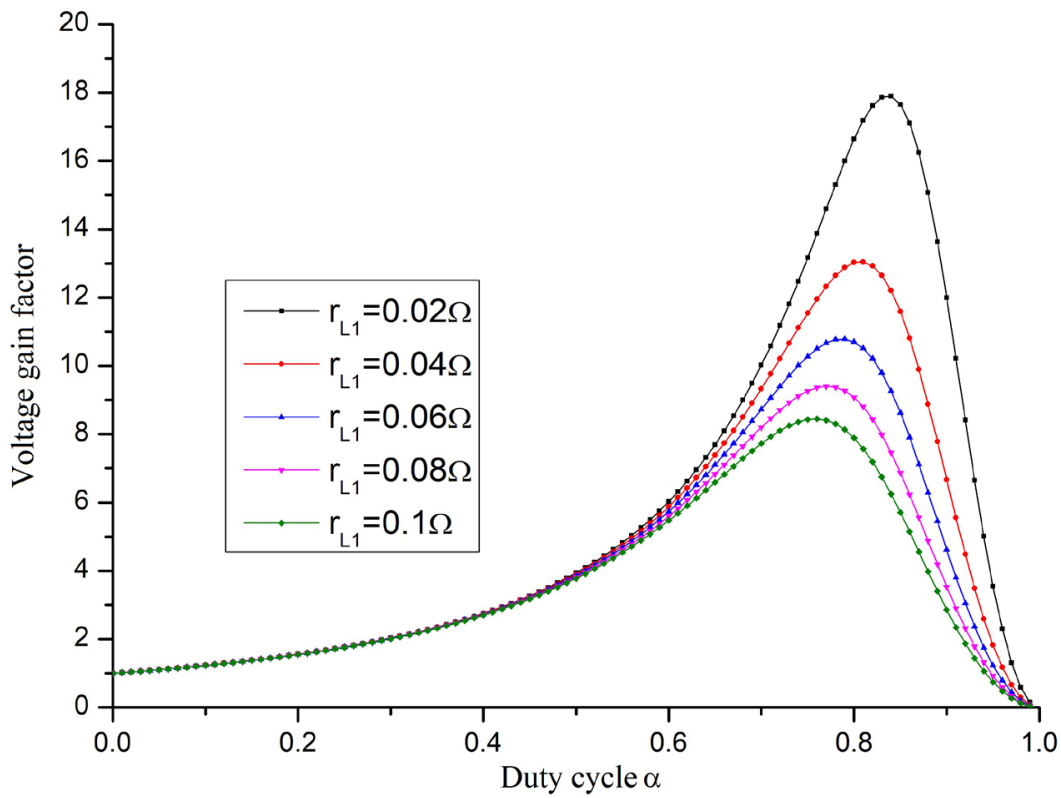

(a)

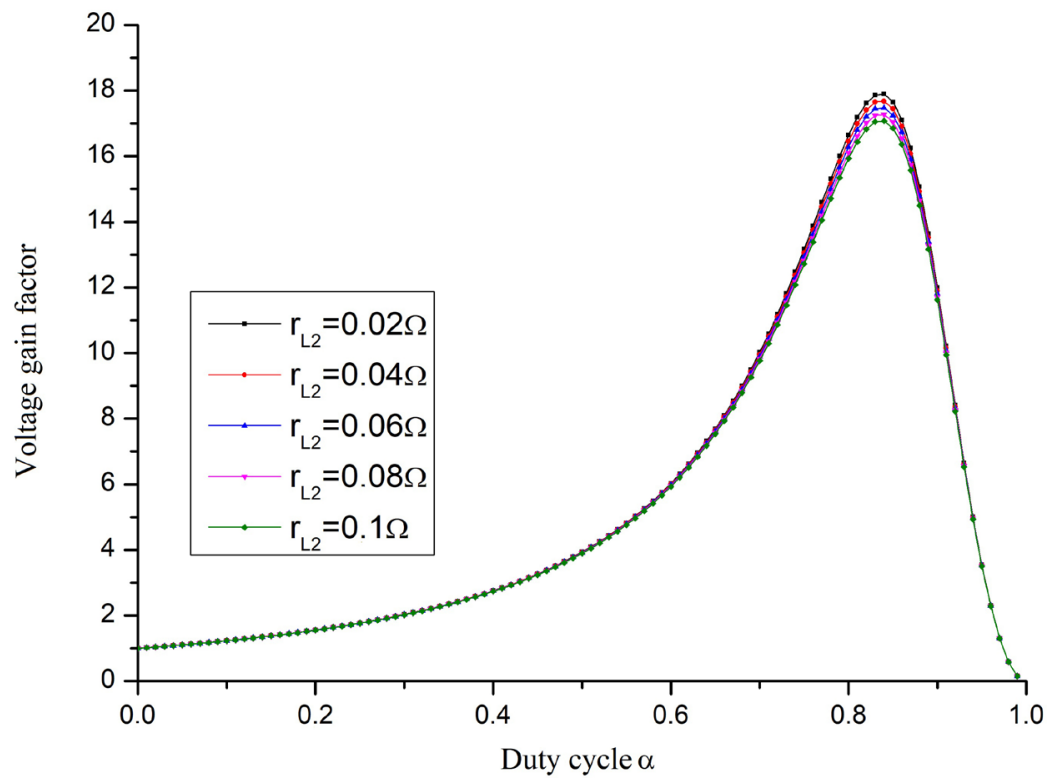

(b)

Figure 3. Voltage gain factor versus duty cycle for various inductor's series resistance values. (a) $r_{L_{2}}=r_{C_{1}}=r_{C_{2}}=0.02 \Omega$; (b) $r_{L_{1}}=r_{C_{1}}=r_{C_{2}}=0.02 \Omega$. 
decreasing for duty cycle approaching unity. We can see that this threshold depend directly on the series resistance value $r_{L}$. For low $r_{L}$ the threshold is reached very close to duty cycle equal to unity but for increasing $r_{L}$ the threshold is reached far from duty cycle equal to unity. This means that the maximum voltage gain factor (corresponding to a duty cycle $\alpha_{0}$ ) is shifted left as $r_{L}$ increases. As losses in the inductor increase, the voltage gain factor decrease very rapidly [19].

However, we can see that, in Figure 3(a), the difference between the maximum of the curve representing a good quality of $L_{1}$ and that of the poor quality is about $52.78 \%$ which is quite considerable as voltage gain factor losses. While in Figure 3(b) the difference is only about $4.56 \%$ between the maximum of the low and high $r_{L_{2}}$ curve. Thus showing that the quality of the inductor $L_{1}$ has an impact 10 times greater on the voltage gain than that of the inductor $L_{2}$.

This means that in practical design the value of $r_{L}$ must be absolutely known and small, with $L_{1}$ priority over $L_{2}$, otherwise the output voltage could not be guaranteed.

We plotted in Figure 4 the voltage gain factor $G$ versus duty cycle $\alpha$ for various $r_{C_{1}}$ in Figure 4(a) and various $r_{C_{2}}$ in Figure 4(b) [18].

These two figures illustrate the effect of the capacitor series resistance on the voltage gain factor. We can see that the quality of the capacitor $C_{1}$ has a greater impact in Figure 4(a) than the capacitor $C_{2}$ in Figure 4(b). In fact, we can loose up to $18.8 \%$ of voltage gain factor for a bad quality of the capacitor $C_{1}$ against $0.64 \%$ for a bad quality of the capacitor $C_{2}$.

That is, the low values of the internal resistances of inductances and capacitors allow us to have a better voltage gain. However, the $r_{L}$ values affect the value of the voltage gain more than the $r_{C}$ values. But different influences are more marked by the values of $r_{L_{1}}$ and the values of $r_{C_{1}}$; the variations of $r_{C_{1}}$ impact more than the variations of $r_{L_{2}}$.

\subsection{Conversion Efficiency}

The challenges to be raised at the level of the converters being not limited to the voltage gain but also to the yield, let us analyze now the conversion efficiency versus the duty cycle.

Figure 5 shows the conversion efficiency profile versus duty cycle for various $r_{L_{1}}$ values in Figure 5(a) and various $r_{L_{2}}$ values in Figure 5(b) [18].

This figure shows that as the duty cycle increases, the conversion decrease and this decrease is very marked for duty cycles close to unity if the series resistance $r_{L}$ of the inductor is small. When $r_{L}$ is high, the losses in the inductor prevail as duty cycle increase leading to the observed decrease of the conversion efficiency [19].

Indeed, we see with the figure that this decrease is very steep for duty cycles close to the unit when the series resistance $r_{L_{1}}$ is weak. When, on the other hand, it is high, the losses in the inductance take precedence over the effects of 
the duty cycle, resulting in a less gross decrease in conversion efficiency but earlier. However, we can see at the level of the figure that the various curves are barely perceptible thus showing the very low impact that the quality of the $L_{2}$ inductance has on the conversion efficiency.

In Figure 6 the Conversion efficiency profile is presented versus duty cycle for various $r_{C_{1}}$ values (Figure 6(a)) and larger $r_{C_{2}}$ (Figure 6(b)).

As observed before (Figure 4), we can see in Figure 6 that the effect of the series

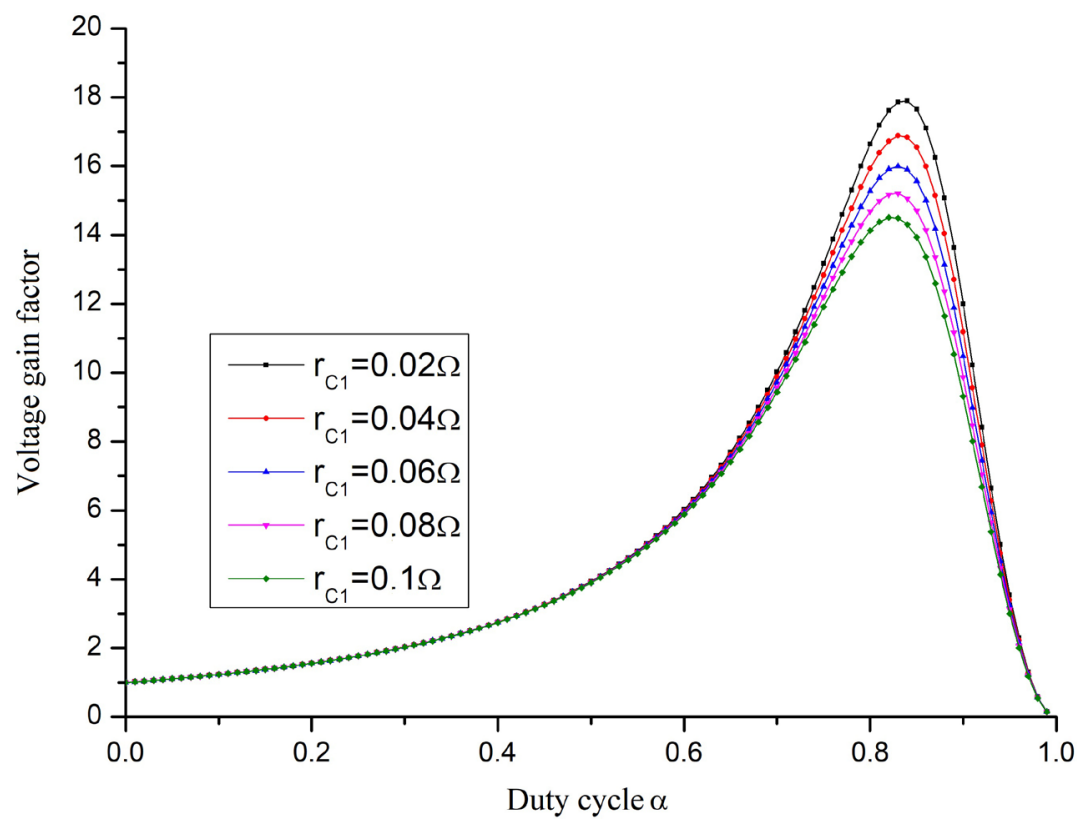

(a)

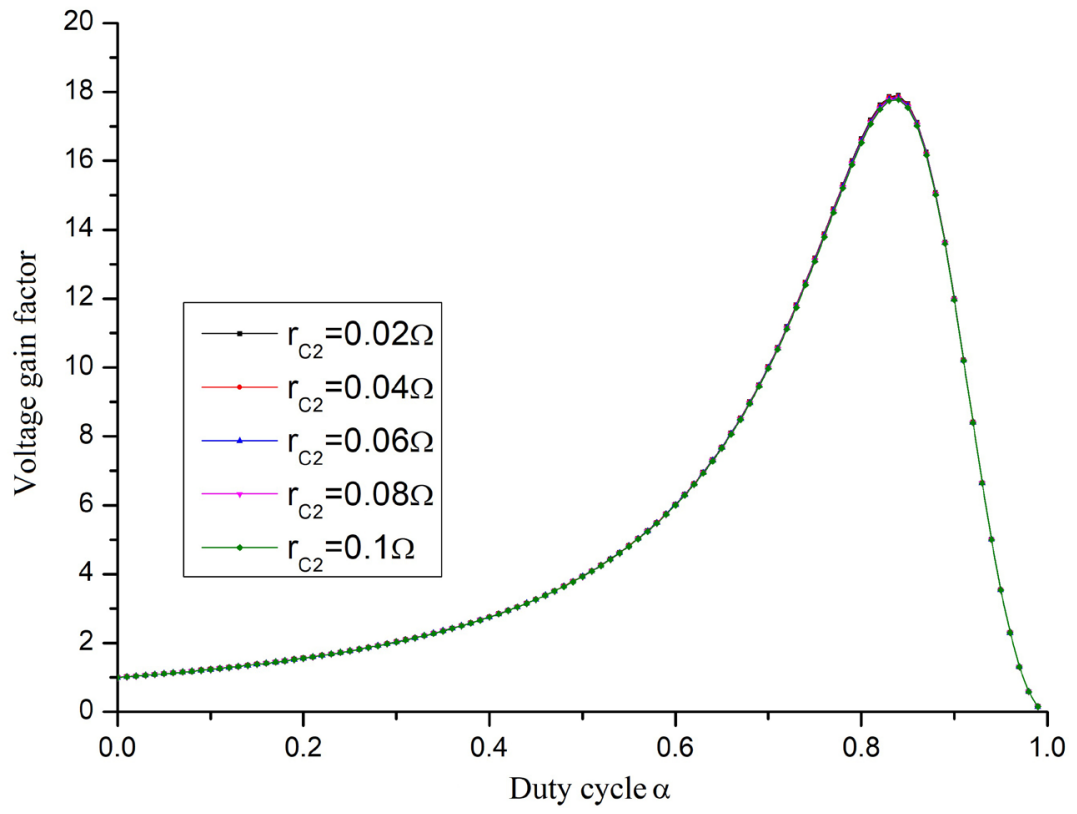

(b)

Figure 4. Voltage gain factor versus duty cycle for various capacitor's series resistance values. (a) $r_{L_{1}}=r_{L_{2}}=r_{C_{2}}=0.02 \Omega$; (b) $r_{L_{1}}=r_{L_{2}}=r_{C_{1}}=0.02 \Omega$. 


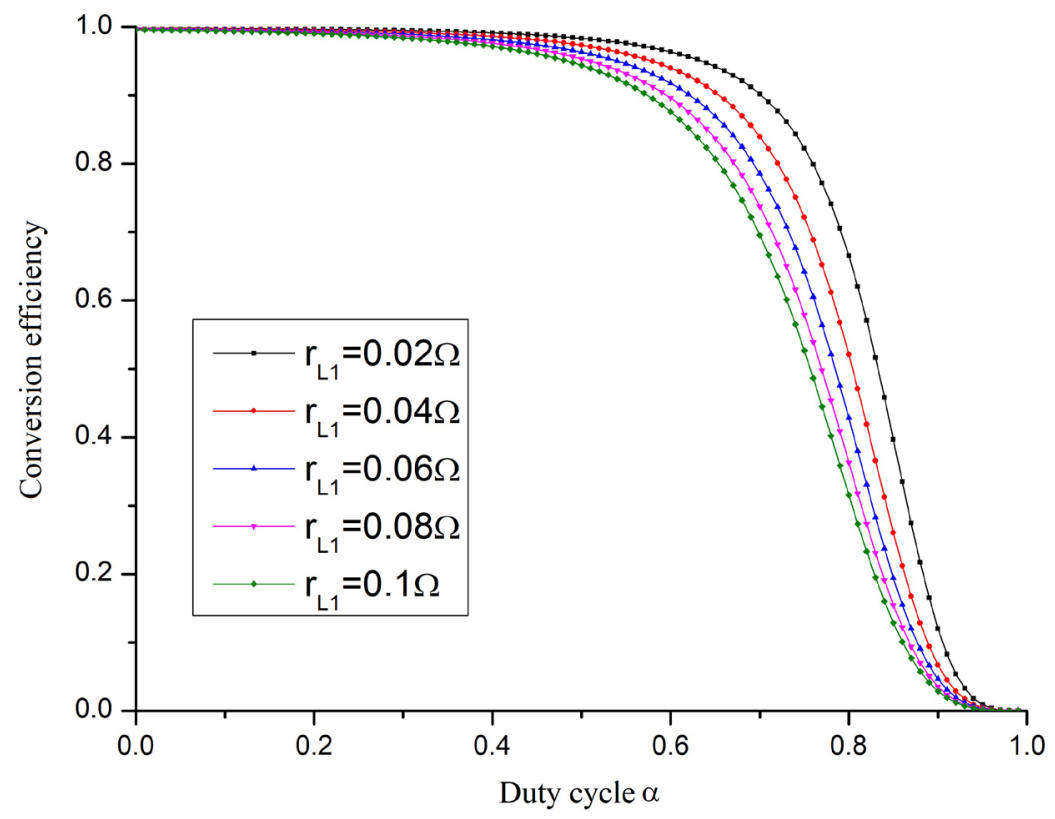

(a)

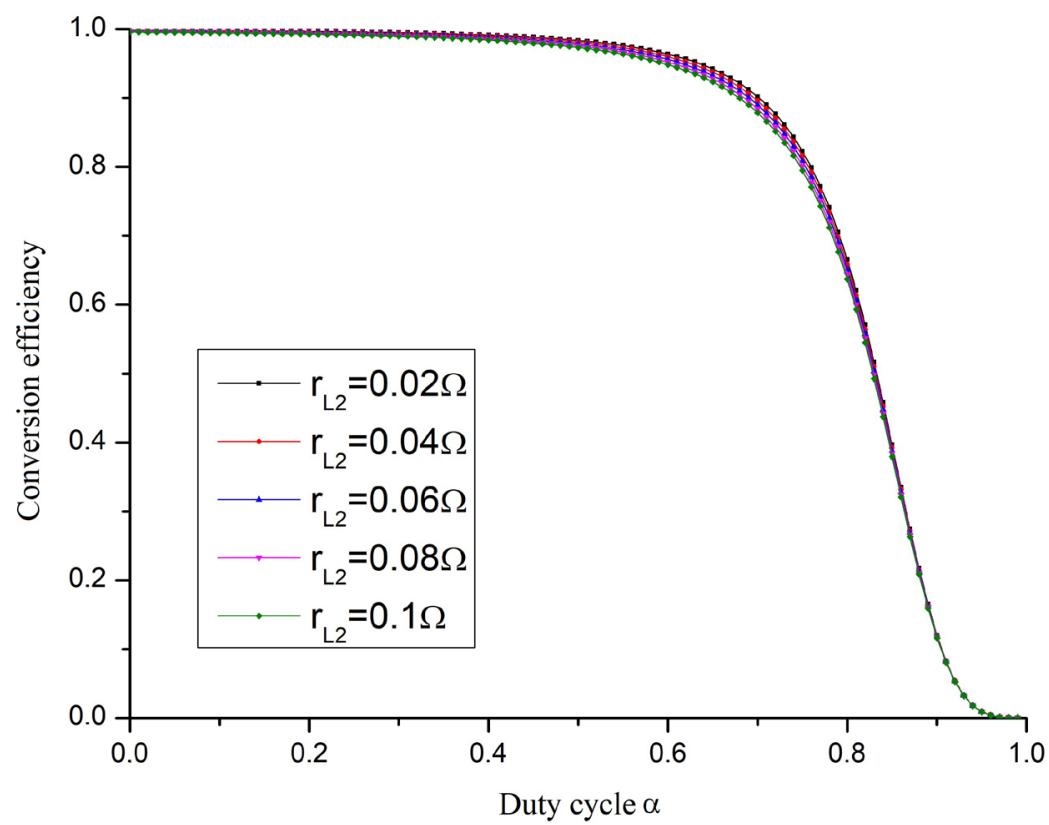

(b)

Figure 5. Conversion efficiency versus duty cycle for various inductor's series resistance values. (a) $r_{L_{2}}=r_{\mathrm{C}_{1}}=r_{\mathrm{C}_{2}}=0.02 \Omega$; (b) $r_{L_{1}}=r_{\mathrm{C}_{1}}=r_{\mathrm{C}_{2}}=0.02 \Omega$.

resistance of the capacitor $C_{2}$ is smaller than that of the series resistance of the capacitor $C_{1}$.

As with the voltage gain curves, we see that the values of $r_{L_{1}}$ influence more on the performance followed by those of $r_{C_{1}}$, then from $r_{L_{2}}$ and then $r_{C_{2}}$.

For low duty cycle, the gain and efficiency curves are inversely proportional but the effects of the internal resistances of inductances and capacitors are identical. These effects predominate in duty cycle neighboring to the unit to the 


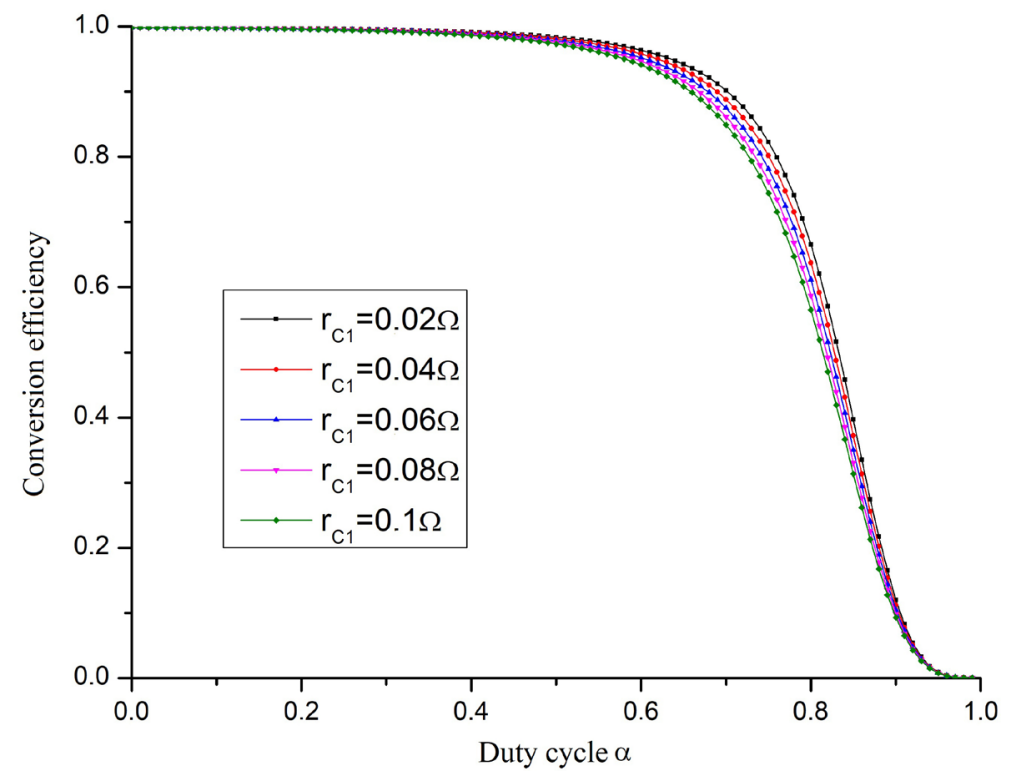

(a)

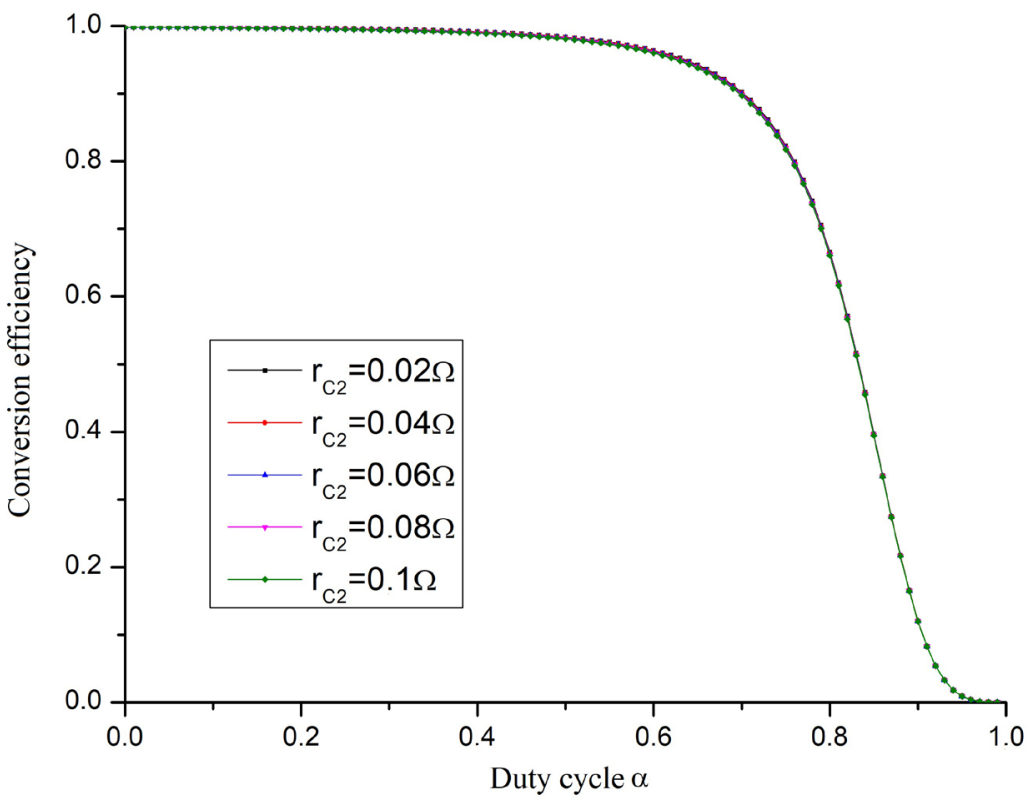

(b)

Figure 6. Conversion efficiency versus duty cycle for various capacitor's series resistance values. (a) $r_{L_{1}}=r_{L_{2}}=r_{C_{2}}=0.02 \Omega$; (b) $r_{L_{1}}=r_{L_{2}}=r_{C_{1}}=0.02 \Omega$.

point of canceling the values of these different curves.

It would be advisable not to operate at duty cycles greater than 0.8 because the effects of series resistances are more marked in this area. Indeed, working with a duty cycle close to the unit increases the stress of the semiconductors and decreases sharply the life of the converter [19].

\section{Conclusions}

We have presented in this paper a detailed theoretical study of the quadratic 
boost converter. We have taken into account the real behavior of the passive components of this converter and we have analyzed its voltage gain factor and conversion efficiency. It has been shown that the quality of the passive components of a converter also plays an important role in the quality of the conversion. Indeed, the deterioration of these components over time will cause underperformance of the converter. However, not all passives components affect the converter in the same way. Input inductor $L_{1}$ must be of the best possible quality as input capacitor $C_{1}$. The output inductor $L_{2}$ has little effect on the performance of the converter contrary to $L_{1}$. Finally, we have the output capacitor $C_{2}$ that has a very low impact on the voltage gain and on the efficiency but remains important for the quality of the output voltage.

We also show that it is not recommended to use duty cycle close to unity [14] [15] because losses effects are most important there with a markedly decrease of both voltage gain factor and conversion efficiency. Next steps are dedicated to building a prototype converter for validation purpose and taking into account switching losses.

\section{Acknowledgements}

We would like to express our gratitude to ISP, University of Uppsala, Sweden, for its support of project BUF01.

\section{Conflicts of Interest}

The authors declare no conflicts of interest regarding the publication of this paper.

\section{References}

[1] Wester, G.W. (1972) Low-Frequency Characterization of Switched DC-DC Converters. Ph.D. Thesis, California Institute of Technology, Pasadena. https://doi.org/10.7907/RMBC-4961

[2] Wester, G.W. and Middlebrook, R.D. (1973) Low-Frequency Characterization of Switched DC-to-DC Converters. IEEE Transactions on Aerospace and Electronic Systems, AES-9, 376-385. https://doi.org/10.1109/TAES.1973.309723

[3] Owen, H.A., Capel, A. and Ferrante, J.G. (1976) Simulation and Analysis Methods for Sampled Power Electronic Systems. Proceedings of the 1976 IEEE Power Electronics Specialists Conference, Cleveland, 8-10 June, 45-55. https://doi.org/10.1109/PESC.1976.7072897

[4] Lee, F.C.Y., Iwens, R.P., Yu, Y. and Triner, J.E. (1979) Generalized Computer-Aided Discrete Time Domain Modeling and Analysis of DC-DC Converters. IEEE Transactions on Industrial Electronics and Control Instrumentation, IECI-26, 58-69. https://doi.org/10.1109/TIECI.1979.351572

[5] Cuk, S. and Middlebrook, R.D. (1977) A General Unified Approach to Modelling Switching Dc-to-Dc Converters in Discontinuous Conduction Mode. Proceedings of the IEEE Power Electronics Specialists Conference, Palo Alto, 14-16 June, 36-57. https://doi.org/10.1109/PESC.1977.7070802

[6] Vorperian, V. and Cuk, S. (1983) Small Signal Analysis of Resonant Converters. 
Proceedings of the IEEE Power Electronics Specialists Conference, Albuquerque, 6-9 June, 269-282. https://doi.org/10.1109/PESC.1983.7069866

[7] Vorperian, V., Tymerski, R. and Lee, F.C.Y. (1989) Equivalent Circuit Models for Resonant and PWM Switches. IEEE Transactions on Power Electronics, 4, 205-214. https://doi.org/10.1109/63.24905

[8] Vorperian, V. (1990) Simplified Analysis of PWM Converters Using Model of PWM Switch: Continuous Conduction Mode. IEEE Transactions on Aerospace and Electronic Systems, 26, 490-496. https://doi.org/10.1109/7.106126

[9] Sanders, S.R., Noworolski, J.M., Liu, X.Z. and Verghese, G.C. (1991) Generalized Averaging Method for Power Conversion Circuits. IEEE Transactions on Power Electronics, 6, 251-259. https://doi.org/10.1109/63.76811

[10] Noworolski, J.M. and Sanders, S.R. (1991) Generalized In-Plane Circuit Averaging. APEC91: Sixth Annual Applied Power Electronics Conference and Exhibition, Dallas, 10-15 March 1991, 445-451. https://doi.org/10.1109/APEC.1991.146212

[11] Lynch, Brian T. Under the Hood of a DC/DC Boost Converter, Texas Instruments Power Supply Design Seminar SEM1800, Topic 3, SLUP284.

https://www.ti.com/power-management/support-training.html

[12] Lopez-Santos, O. (2015) Contribution à l'étude de la conversion DC-AC dans des systèmes photovoltaïques: Convertisseurs orientés au module PV. Automatique/ Robotique. Institut National Des Sciences Appliquées De Toulouse (INSA Toulouse), Français.

[13] Surya, P.B. and Ramprasath, M.S. (2018) Mathematical Modelling and Performance Analysis of Quadratic Boost Converter. International Journal of Scientific \& Engineering Research, 9, 190-196.

[14] Choudhury, T.R. and Nayak, B. (2015) Comparison and Analysis of Cascaded and Quadratic Boost Converter. IEEE Power, Communication and Information Technology Conference (PCITC), Siksha 'O' Anusandhan University, Bhubaneswar, 15-17 October 2015, 78-83. https://doi.org/10.1109/PCITC.2015.7438108

[15] Vite, P.M.G., Rangel, C.A.S., Caro, J.C.R. and David, F.M. (2017) A DC-DC Converter with Quadratic Gain and Input Current Ripple Cancelation at a Selectable Duty Cycle. Renewable Energy, 101, 431-436.

http://dx.doi.org/10.1016/j.renene.2016.09.010

[16] Durán, E., Andújar, J.M., Segura, F. and Barragán, A. J. (2011) A High-Flexibility DC Load for Fuel Cell and solar Arrays Power Sources Based on DC-DC Converters. Applied Energy, 88, 1690-1702. https://doi.org/10.1016/j.apenergy.2010.11.002

[17] Tattiwong, K. and Bunlaksananusorn, C. (2014) Analysis Design and Experimental Verification of a Quadratic Boost Converter. TENCON 2014-2014 IEEE Region 10 Conference, Bangkok, 29 January 2015, 1-6. https://doi.org/10.1109/TENCON.2014.7022467

[18] Honadia, P.A.A. (2018) Contribution to the Improvement of Photovoltaic Systems Connected to the Grid. Ph.D. Thesis, Cheikh Anta Diop University, Dakar.

[19] Honadia, P.A.A., Barro, F.I. and Sané, M. (2018) Performance Analysis of a Boost Converter with Components Losses. Energy and Power Engineering, 10, 399-413. https://doi.org/10.4236/epe.2018.109025 


\section{Appendix}

The RMS values of the currents in the inductances $L_{1}$ and $L_{2}$ of this quadratic elevator are respectively $I_{\text {eff } L_{1}}$ and $I_{\text {eff } L_{L_{2}}}$ whose expressions are:

$$
I_{\text {eff }}=I_{L_{1}}=\frac{I_{0}}{(1-\alpha)^{2}}
$$

and

$$
I_{\text {eff } L_{2}}=I_{L_{2}}=\frac{I_{0}}{(1-\alpha)}
$$

and the losses in the inductors are therefore given by:

$$
\begin{gathered}
P_{r_{L_{1}}}=r_{L_{1}} \cdot I_{\text {eff } L_{L_{1}}}^{2} \\
P_{r_{L_{1}}}=r_{L_{1}} \cdot \frac{I_{0}^{2}}{(1-\alpha)^{4}}
\end{gathered}
$$

and

$$
\begin{gathered}
P_{r_{L_{2}}}=r_{L_{2}} \cdot I_{\text {eff } L_{2}}^{2} \\
P_{r_{L_{2}}}=r_{L_{2}} \cdot \frac{I_{0}^{2}}{(1-\alpha)^{2}} \\
P_{r_{L}}=P_{r_{L_{1}}}+P_{r_{L_{2}}}=r_{L_{1}} \cdot I_{\text {eff } L_{1}}^{2}+r_{L_{2}} \cdot I_{\text {eff } L_{2}}^{2} \\
P_{r_{L}}=r_{L_{1}} \cdot \frac{I_{0}^{2}}{(1-\alpha)^{4}}+r_{L_{2}} \cdot \frac{I_{0}^{2}}{(1-\alpha)^{2}}
\end{gathered}
$$

It is the same for capacitors whose expressions of the currents flowing through them are given by:

$$
\begin{gathered}
I_{C_{1}}= \begin{cases}-I_{L_{2}}=-\frac{I_{0}}{1-\alpha} & \text { pour } 0<t \leq \alpha T \\
I_{L_{1}}-I_{L_{2}}=\frac{I_{0} \alpha}{(1-\alpha)^{2}} & \text { pour } \alpha T<t \leq T\end{cases} \\
I_{C_{2}}= \begin{cases}-I_{0} & \text { pour } 0<t \leq \alpha T \\
I_{L_{2}}-I_{0}=\frac{I_{0} \alpha}{(1-\alpha)} & \text { pour } \alpha T<t \leq T\end{cases}
\end{gathered}
$$

The RMS values of the currents are therefore obtained by:

$$
\begin{gathered}
I_{\text {eff }_{C_{1}}}=\sqrt{\frac{1}{T} \int_{0}^{T} I_{C_{1}}^{2} \mathrm{~d} t}=\sqrt{\frac{1}{T}\left[\int_{0}^{D t} \frac{I_{0}^{2}}{(1-\alpha)^{2}} \mathrm{~d} t+\int_{D T}^{T} \frac{I_{0}^{2} \alpha^{2}}{(1-\alpha)^{4}} \mathrm{~d} t\right]} \\
I_{\text {eff }_{C_{1}}}=\sqrt{\frac{1}{T}\left\{\frac{I_{0}^{2}}{(1-\alpha)^{2}} \cdot \alpha T+\frac{I_{0}^{2} \alpha^{2}}{(1-\alpha)^{4}} \cdot(T-\alpha T)\right\}}
\end{gathered}
$$

This gives by calculation: 


$$
I_{\text {eff } C_{1}}=\frac{I_{0}}{(1-\alpha)^{2}} \sqrt{\alpha(1-\alpha)}
$$

and

$$
\begin{aligned}
I_{\text {eff } C_{2}} & =\sqrt{\frac{1}{T} \int_{0}^{T} I_{C_{2}}^{2} \mathrm{~d} t}=\sqrt{\frac{1}{T}\left[\int_{0}^{\alpha t} I_{0}^{2} \mathrm{~d} t+\int_{\alpha T}^{T} \frac{I_{0}^{2} \alpha^{2}}{(1-\alpha)^{2}} \mathrm{~d} t\right]} \\
& =\sqrt{\frac{1}{T}\left\{I_{0}^{2} \alpha T+\frac{I_{0}^{2} \alpha^{2}}{(1-\alpha)^{2}} \cdot(T-\alpha T)\right\}}
\end{aligned}
$$

giving by calculation:

$$
I_{\text {eff } C_{2}}=I_{0} \sqrt{\frac{\alpha}{1-\alpha}}
$$

Capacitor losses are:

$$
\begin{gathered}
P_{r_{C}}=P_{r_{C_{1}}}+P_{r_{C_{2}}} \\
P_{r_{C}}=r_{C_{1}} \cdot I_{\text {eff }_{C_{1}}}^{2}+r_{C_{2}} \cdot I_{e^{2} f_{C_{2}}}^{2} \\
P_{r_{C}}=r_{C_{1}} \cdot \frac{I_{0}^{2} \alpha(1-\alpha)}{(1-\alpha)^{4}}+r_{C_{2}} \cdot \frac{I_{0}^{2} \alpha}{(1-\alpha)}
\end{gathered}
$$

The total power loss of the converter is therefore:

$$
\begin{gathered}
P_{L S}=P_{r_{L_{1}}}+P_{r_{L_{2}}}+P_{r_{C_{1}}}+P_{r_{C_{2}}} \\
P_{L S}=r_{L_{1}} \cdot I_{e f f L_{1}}^{2}+r_{L_{2}} \cdot I_{e f L_{L_{2}}}^{2}+r_{C_{1}} \cdot I_{\text {eff } C_{1}}^{2}+r_{C_{2}} \cdot I_{e f f C_{2}}^{2} \\
\Rightarrow P_{L S}=r_{L_{1}} \cdot \frac{I_{0}^{2}}{(1-\alpha)^{4}}+r_{L_{2}} \cdot \frac{I_{0}^{2}}{(1-\alpha)^{2}}+r_{C_{1}} \cdot \frac{I_{0}^{2} \cdot \alpha(1-\alpha)}{(1-\alpha)^{4}}+r_{C_{2}} \cdot \frac{I_{0}^{2} \cdot \alpha}{(1-\alpha)}
\end{gathered}
$$

By replacing $I_{I}$ with $I_{L_{1}}=\frac{I_{0}}{(1-\alpha)^{2}}$ the expression becomes:

$$
\begin{aligned}
V_{I} \cdot \frac{I_{0}}{(1-\alpha)^{2}}= & V_{0} \cdot I_{0}+r_{L_{1}} \cdot \frac{I_{0}^{2}}{(1-\alpha)^{4}}+r_{L_{2}} \cdot \frac{I_{0}^{2}}{(1-\alpha)^{2}} \\
& +r_{C_{1}} \cdot \frac{I_{0}^{2} \cdot \alpha(1-\alpha)}{(1-\alpha)^{4}}+r_{C_{2}} \cdot \frac{I_{0}^{2} \cdot \alpha}{(1-\alpha)}
\end{aligned}
$$

This gives a calculated expression of the voltage gain $\left(G_{r_{L}, r_{C}}\right)$ of the converter with the inductive and capacitive ESR effects.

$$
G_{r_{L}, r_{C}}=\frac{V_{0}}{V_{I}}=\frac{(1-\alpha)^{2} R}{r_{L_{1}}+(1-\alpha)^{2} \cdot r_{L_{2}}+\alpha(1-\alpha) \cdot r_{C_{1}}+\alpha(1-\alpha)^{3} \cdot r_{C_{2}}+(1-\alpha)^{4} R}
$$

We obtain the expression of the efficiency of the converter by the relation:

$$
\begin{gathered}
\eta=\frac{P_{0}}{P_{I}} \\
\eta=\frac{P_{0}}{P_{0}+P_{L S}}
\end{gathered}
$$


where $P_{L S}$ : designates the overall power loss in the quadratic boost system. $P_{I}$ and $P_{0}$ designating respectively the input and output powers.

$$
\begin{gathered}
P_{I}=P_{0}+P_{r_{L_{1}}}+P_{r_{L_{2}}}+P_{r_{C_{1}}}+P_{r_{C_{2}}} \\
=V_{0} \cdot I_{0}+r_{L_{1}} \cdot I_{\text {eff } L_{1}}^{2}+r_{L_{2}} \cdot I_{\text {eff } L_{2}}^{2}+r_{C_{1}} \cdot I_{\text {eff } C_{1}}^{2}+r_{C_{2}} \cdot I_{\text {eff } C_{2}}^{2} \\
\Rightarrow V_{I} \cdot I_{I}=V_{0} \cdot I_{0}+r_{L_{1}} \cdot \frac{I_{0}^{2}}{(1-\alpha)^{4}}+r_{L_{2}} \cdot \frac{I_{0}^{2}}{(1-\alpha)^{2}} \\
+r_{C_{1}} \cdot \frac{I_{0}^{2} \cdot \alpha(1-\alpha)}{(1-\alpha)^{4}}+r_{C_{2}} \cdot \frac{I_{0}^{2} \cdot \alpha}{(1-\alpha)} \\
\text { or } P_{0}=V_{0} \cdot I_{0} \\
\text { and } V_{0}=R_{L} \cdot I_{0} \\
\Rightarrow P_{L S}=r_{L_{1}} \cdot \frac{I_{0}^{2}=\frac{P_{0}}{R}}{R(1-\alpha)^{4}}+r_{L_{2}} \cdot \frac{P_{0}}{R(1-\alpha)^{2}}+r_{C_{1}} \cdot \frac{P_{0} \cdot \alpha(1-\alpha)}{R(1-\alpha)^{4}}+r_{C_{2}} \cdot \frac{P_{0} \cdot \alpha}{R(1-\alpha)} \\
\Rightarrow P_{L S}=\left[\frac{r_{L_{1}}+\alpha(1-\alpha) \cdot r_{C_{1}}}{(1-\alpha)^{4} \cdot R}+\frac{r_{L_{2}}+\alpha(1-\alpha) \cdot r_{C_{2}}}{(1-\alpha)^{2} \cdot R}\right] \cdot P_{0} \\
\eta_{r_{L}, r_{C}}=\frac{P_{0}}{P_{0}\left[1+\frac{P_{L S}}{P_{0}}\right]} \\
\eta_{r_{L}, r_{C}}=\frac{1}{1+\frac{P_{L S}}{P_{0}}}
\end{gathered}
$$

This finally gives:

$$
\eta_{r_{L}, r_{C}}=\frac{1}{1+\left[\frac{r_{L_{1}}+\alpha(1-\alpha) \cdot r_{C_{1}}}{(1-\alpha)^{4} \cdot R}+\frac{r_{L_{2}}+\alpha(1-\alpha) \cdot r_{C_{2}}}{(1-\alpha)^{2} \cdot R}\right]}
$$

